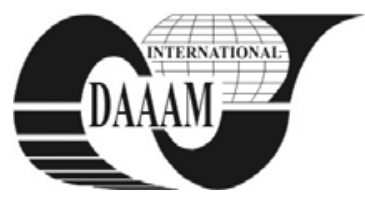

\title{
RESEARCH ON IRON AND NICKEL MIGRATION FROM AUSTENITIC STAINLESS STEELS INTO ACID FOOD PRODUCTS
}

\section{STROE, S[ilviu] - G[abriel]; GUTT, G[heorghe]; GUTT, S[onia]; POROCH - SERITAN, M[aria]; SEVERIN, T[raian] - L[ucian] \& MAIORESCU, M[irela]}

\begin{abstract}
The aim of this paper consists in studying the migration phenomenon of iron and nickel ions from austenitic stainless steel used in food industry. The study on metallographic structure has shown uniform distribution of the constitutive elements, reducing significantly the possibility for galvanic corrosion phenomenon to occur. The chemical composition of material is determined by EDX-Bruker microprobe. The contact between the metallic material and food product is simulated by using a solution of 3\% acetic acid, the samples being kept for different periods of time at the temperature of $100^{\circ} \mathrm{C}$. As a result of the analyses made by atomic absorption spectrometry, some strong dependency between iron and nickel concentrations found in food stimulant, processing degree of surfaces and contact time between the two media has been noticed.
\end{abstract}

Key words: roughness, simulant, migration, iron, nickel

\section{INTRODUCTION}

Metallic materials meant to get in contact with food products while processing are used in a wide range of goods, from equipment parts, conveyer pipe lines, to tanks and containers used in preserving food products. Stainless steels are met in many processing activities of food raw materials due to their resistance to corrosion in different media and their mechanical properties as compared with other metallic materials. From this point of view, austenitic stainless steels such as AISI 304 and AISI 316, having chrome as main alloying element, minimum content of $12 \%$ and nickel between 8-10.5 \% are the most used materials (Council of Europe, 2001). The research made over the last years led to the conclusion that nickel presence in these alloys increases resistance to corrosion by reducing the migration rate, though the migration of nickel ions into food products has some negative influence on human health (Herting et al., 2007a).

The daily intake regulated by legislation is of $0.005 \mathrm{mg}$ $\mathrm{Ni} / \mathrm{kg}$ body (WHO, 1997), and daily intake by means of food products is estimated at $0.15-0.7 \mathrm{mg} \mathrm{Ni} /$ day (Codex, 1995).

As regards iron, there are no strict regulations on the maximum admitted limits, though the Common Commission of Experts FAO/WHO established provisorily a maximum admitted dose of $0.8 \mathrm{mg} \mathrm{Fe} / \mathrm{kg}$ bw, and WHO does not recomand a maximum limit for drinking water as the main problem of iron is its deficiency in the human body and not a toxicological one.

Having in view all these aspects regarding the influence of metallic ions present in food products, the aim of this paper is to study the migration of iron and nickel ions from austenitic stainless steels into food products.

\section{MATERIALS AND METHODS}

\subsection{Material composition and sample preparation}

The study is made on an astenitic stainless steel, which structure in Figure 1 and chemical composition in Table 2 are determined by scanning electronic microscopy (SEM) (Vega LMU Tescan) and X-ray microprobe (EDX Bruker microprobe) of the microscope. The surfaces of stainless steel samples getting into contact with the corrosive medium have been processed by metallographic paper of different granularities $(180 \mu \mathrm{m}$ and $800 \mu \mathrm{m})$ to obtain different roughnesses and by felt to obtain glossy surface. Sample cleaning has been made by ultrasounding at the frequency of $45 \mathrm{kHz}$ for 6 minutes with ultrasounding bath Elma TI-H-15.

\subsection{Iron and nickel migration testing}

The study of migration of iron and nickel ions has been made accordingly to the legislative Italian stipulations D.M. 2103-1973 and Romanian norm from the year 2002 regarding the materials and objects that come into contact with foods (Herting et al., 2008).

A solution of acetic acid $3 \%$, simulant meant to replace acid foods (Food Standards Agency, 2003) has been used as corrosive medium. The surface of stainless steel in contact with the simulant is of approximately $16 \mathrm{~cm}^{2}$, and the volume of the simulant used is of $10 \mathrm{ml}$, so that the ratio between the surface of metallic material exposed and solution volume be approximately of 1.6, the ratio value being in the limits $0.5-2$ stipulated by D.M. 21-03-1973. Experiment planning is shown in table1.

\subsection{Spectroscopic analysis of solutions after being exposed}

Iron and nickel concentrations from solutions were determined by flame (air- acetylene) - atomic absorption spectrometry (AAS-6300 Shimadzu) at characteristic wavelengths: $\lambda_{\mathrm{Fe}}=248.3 \mathrm{~nm}$ and $\lambda_{\mathrm{Ni}}=232.0 \mathrm{~nm}$.

The detection limit is of $1.0 \mu \mathrm{g} \cdot \mathrm{L}^{-1}$ for nickel and 15.0 $\mu \mathrm{g} \cdot \mathrm{L}^{-1}$ for iron. All the experiments have been made in the laboratory of foods' quality analysis of the Faculty of Food Engineering within “Stefan cel Mare”University of Suceava.

\begin{tabular}{|c|c|c|c|}
\hline Sample & $\begin{array}{c}\text { Surface } \\
\text { roughness }\end{array}$ & $\begin{array}{c}\text { Exposure time } \\
\text { (min) }\end{array}$ & $\begin{array}{c}\text { Temperature } \\
\left({ }^{\circ} \mathrm{C}\right)\end{array}$ \\
\hline 1 & 180 & 30 & \multirow{15}{*}{100} \\
\hline 2 & 800 & 30 & \\
\hline 3 & polished & 30 & \\
\hline 4 & polished & 45 & \\
\hline 5 & 800 & 45 & \\
\hline 6 & 180 & 45 & \\
\hline 7 & polished & 60 & \\
\hline 8 & 180 & 60 & \\
\hline 9 & 800 & 60 & \\
\hline 10 & polished & 75 & \\
\hline 11 & 800 & 75 & \\
\hline 12 & 180 & 75 & \\
\hline 13 & polished & 90 & \\
\hline 14 & 800 & 90 & \\
\hline 15 & 180 & 90 & \\
\hline
\end{tabular}

Tab. 1. Experiment Planning 


\section{RESULTS AND DISCUSSION}

3.1 Structure and chemical composition of the material

As a result of studying the material chemical composition, one may notice that it is about high chrome and nickel alloyed steel. Resistance to corrosion is strongly related to alloying degree (Table 1); uniform distribution of alloying elements provides steel with good resistance to corrosive media (Council of Europe, 2001).

\subsection{Influence of surface processing degree on iron and nickel migration rate}

The surface finishing degree together with other properties such as surface area in contact, have an important influence on the iron and nickel migration rate from stainless steel (Figure 2 and Figure 3).Mention should be made that in sooth, roughness of metallic surfaces in contact with food products belongs to those used in the samples worked with in the experiment with metallographic paper of $180 \mu \mathrm{m}$ or $800 \mu \mathrm{m}$. Studies have shown that glossy surfaces have low migration rates of metallic ions (iron and nickel) into the simulant solutions for all periods of contact metallic surface/simulant, whereas rough surfaces (processed through granularity $800 \mu \mathrm{m}$ of metallographic paper), which expose a much larger real surface than the geometric one, have very high iron and nickel migration rate. The concentrations of the metal migrated to stimulant, in the case of both metals (iron and nickel) are the lowest in glossy surface and contact time of 30 minutes.

After having studied the values of figure 2 it is obvious that the closest iron migration rates as value, without great differences, for all the three finishing degrees occur when samples are kept in contact with the stimulant for 60 minutes. For the other keeping times, one may see very significant differences between the quantities of iron ions migrated, depending on the surface processing degree. As a result of comparative study on the quantities of iron and nickel ions migrated (Figure 2 and Figure 3) nickel has a much lower migration rate than iron. Nickel is also found in stimulant, the same as iron is, in quantities close to the values when samples have been exposed to acid medium for 60 minutes. The biggest quantity of nickel migrated from stainless steel to food stimulant is noticed when the metallic sample of the highest roughness has been exposed to for 90 minutes.

\section{CONCLUSION}

Iron and nickel migration from austenitic stainless steel has been studied by using as corrosive medium a solution of $3 \%$ acetic acid, simulant recommended by both the legislative regulations D.M. 21-03-1978 and Romanian norm which stipulates the testing conditions of materials coming into contact with foods. As a result of metallographic analysis, good homogeneity of steel is observed, leading to high resistance to corrosion of this material.

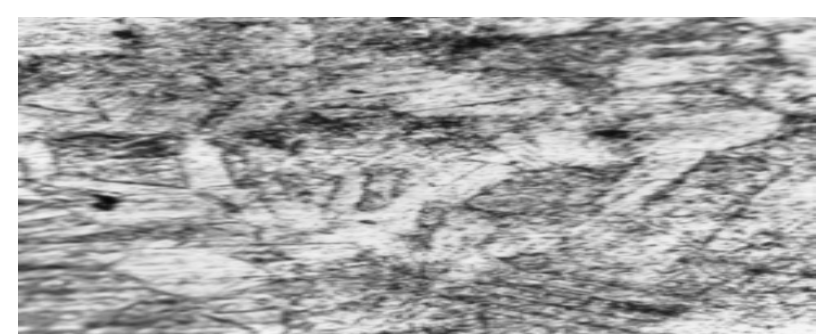

Fig. 1. Microstructure of steel studied (metallographic microscope Optika - magnification 800x)

\begin{tabular}{|c|c|c|c|c|}
\hline $\mathrm{Cr}$ & $\mathrm{Mn}$ & $\mathrm{Ni}$ & $\mathrm{C}$ & $\mathrm{Fe}$ \\
\hline 17.575 & 0.850 & 10.535 & 0.023 & 71.017 \\
\hline
\end{tabular}

Tab. 2. Chemical composition of stainless steel studied (wt \%)

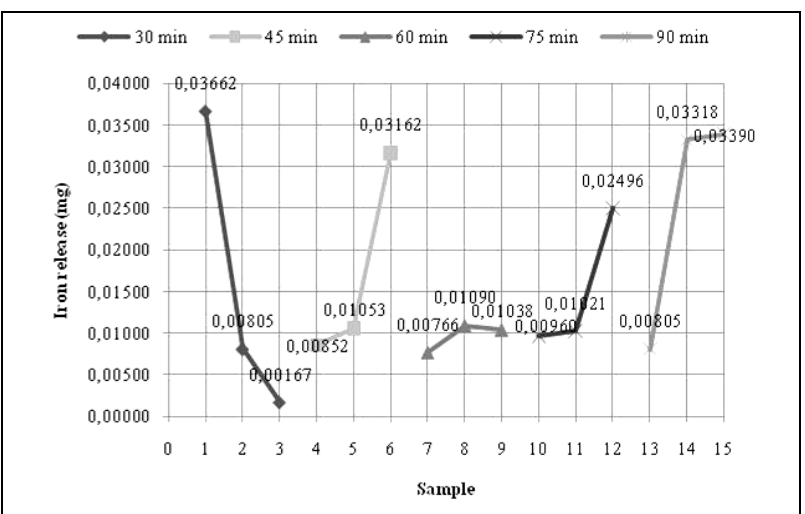

Fig. 2. The influence of surface finishing degree and time contact surface/simulant upon iron migration rate from material

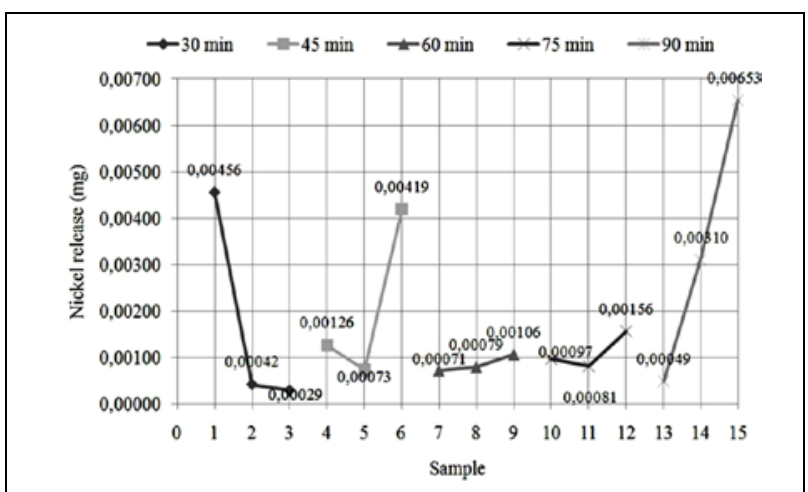

Fig. 3 The influence of surface finishing degree and time contact surface/simulant upon Ni migration rate from material

The results obtained offer an important view on the influence of processing material state and working parameters (chemical composition of surfaces, exposure temperature and size of contact surfaces). The rougher the contact surface with corrosive medium, the higher the migration rate is, differences of few higher orders of magnitude being noticed. A very small difference between the quantities transferred to solution may be observed both in iron and nickel, in the case of exposure time of 60 de minutes, irrespective of the surface processing state. This phenomenon is interesting to be further studied on. The study shows a strong influence of technological parameters upon the migration rate of metallic ions from stainless steel to foods. Research will be further carried on for other metals, too (chrome, manganese, cobalt) as well as under other conditions (different temperature, other simulants).

\section{REFERENCES}

Codex Alimentarius Commission (1995). Document no. CX/FAC 96/17. Join FAO/WHO food standards programme. Codex general standard for contaminants and toxins in foods. Directive 91/338/EEC, pp. 59

Council of Europe (2001). Technical Document. Guidelines on metals and alloys used as food contact materials

Food Standards Agency (2003). Migration test protocols for electroplated and dipped metalware intended for food cantact

Herting, G., Odnevall Wallinder, I., Leygraf, C. (2007a). Metal release from various grades of stainless steel exposed $t i$ synthetic body fluids, Corros. Sci., no. 49, pp.103-111

Herting, G., Odnevall Wallinder, I., Leygraf, C. (2008). Corrosion-induced release of chromium and iron from ferritic stainless steel grade AISI 430 in simulated food contact, Journal of Food Engineering 87, pp. 291-300 